\title{
Decoding P300 Variability Using Convolutional Neural Networks
}

\author{
Amelia J. Solon ${ }^{1,2 *}$, Vernon J. Lawhern ${ }^{1}$, Jonathan Touryan ${ }^{1}$, Jonathan R. McDaniel ${ }^{1,2}$, \\ Anthony J. Ries ${ }^{1}$ and Stephen M. Gordon ${ }^{1,2}$ \\ ${ }^{1}$ Human Research and Engineering Directorate, U.S. Army Research Laboratory, Adelphi, MD, United States, ${ }^{2}$ DCS \\ Corporation, Alexandria, VA, United States
}

\section{OPEN ACCESS}

Edited by:

Hasan Ayaz,

Drexel University, United States

Reviewed by:

Mickael Causse,

National Higher School of Aeronautics and Space, France

Jing Jin,

East China University of Science and

Technology, China

Yuanqing $L i$

South China University of Technology,

China

*Correspondence:

Amelia J. Solon

asolon@dcscorp.com

Received: 02 March 2019 Accepted: 28 May 2019

Published: 14 June 2019

Citation:

Solon AJ, Lawhern VJ, Touryan J, McDaniel JR, Ries AJ and Gordon SM

(2019) Decoding P300 Variability

Using Convolutional Neural Networks.

Front. Hum. Neurosci. 13:201.

doi: 10.3389/fnhum.2019.00201
Deep convolutional neural networks $(\mathrm{CNN})$ have previously been shown to be useful tools for signal decoding and analysis in a variety of complex domains, such as image processing and speech recognition. By learning from large amounts of data, the representations encoded by these deep networks are often invariant to moderate changes in the underlying feature spaces. Recently, we proposed a CNN architecture that could be applied to electroencephalogram (EEG) decoding and analysis. In this article, we train our CNN model using data from prior experiments in order to later decode the P300 evoked response from an unseen, hold-out experiment. We analyze the CNN output as a function of the underlying variability in the $\mathrm{P} 300$ response and demonstrate that the CNN output is sensitive to the experiment-induced changes in the neural response. We then assess the utility of our approach as a means of improving the overall signal-to-noise ratio in the EEG record. Finally, we show an example of how CNN-based decoding can be applied to the analysis of complex data.

Keywords: neural decoding, EEG, deep learning, convolutional neural network, P300

\section{INTRODUCTION}

Decades of neuroscience research have yielded profound insights into how the brain processes stimuli, integrates perceptual information, adapts to dual-task demands, and coordinates behavior. Using high-resolution electroencephalogram (EEG), researchers have examined the time course of multiple neural responses, including those related to stimulus encoding, cognitive processes, error detection, and motor movement. However, despite the extensive amount of research into such phenomena and the development of advanced imaging systems and signal processing techniques, the preferred method of analysis is still to collect large numbers of precisely timed trials within controlled experimental paradigms. This preference is due in part to the low signal-to-noise ratio (SNR) of task-relevant neural activity within the EEG record.

To improve the SNR of both oscillatory and evoked EEG signals, several neural decoding approaches have been developed. These approaches include methods such as Independent Component Analysis (ICA), Hierarchical Discriminant Component Analysis (HDCA), and Common Spatial Patterns (CSP), among others (Jung et al., 2000; Parra et al., 2005, 2008; Lemm et al., 2011). Whether these techniques are utilized for signal processing (e.g., ICA), or machine learning applications (e.g., CSP), they are calibrated using subject-specific training data. Such a requirement, however, reinforces the need for a large number of trials to reduce the likelihood of overfitting during the training process. Furthermore, these approaches make a number of assumptions about the data, such as non-Gaussianity, stationarity, whiteness, or statistically independent unitary dimensions, which may not extend to cross-session, cross-subject, 
or cross-experiment analyses. For example, ICA, CSP, and HDCA all utilize some form of spatial filtering, which can quickly become sub-optimal with slight variations in EEG electrode placement, as occurs across subjects or recording sessions (Marathe et al., 2016). ICA can converge on differing solutions for the same subject over different temporal epochs (Hsu et al., 2018) or fail to capture signal variance due to enforcement of statistical independence (Gordon et al., 2015). In short, we are not aware of any neural decoding approaches that can be robustly applied across datasets or experimental paradigms, such that they do not require subject-, or at least experiment-, specific training data.

Recent advances in deep convolutional neural networks (CNNs) for EEG analysis (Cecotti and Graser, 2011; Cecotti et al., 2014; Manor and Geva, 2015; Shamwell et al., 2016; Schirrmeister et al., 2017; Lawhern et al., 2018; Roy et al., 2019) have opened the door for development and training of largescale generalized models, i.e., models that can reliably decode neural activity across subjects and experimental configurations. In the broader machine learning community, CNNs have often been applied to pattern recognition problems such as automatic speech recognition and image processing (see Hinton et al., 2012; Schmidhuber, 2014; LeCun et al., 2015 for reviews). CNNs make limited assumptions about the underlying data, can learn from large, diverse datasets, and extrapolate well to previously unseen data. Although generalized neural decoding models, such as those enabled by $\mathrm{CNN}$ architectures, have the potential to interpret data in more complex and less repeatable scenarios, as well as generally enhance SNR, it is important to quantitatively establish the link between the decoding model output and the underlying neural phenomena.

In this paper, we (1) establish such a link for the visual P300 evoked response, and (2) introduce a CNN-based approach for EEG decoding that can alleviate the need for large amounts of the test experiment's data. We validate our CNN approach using a leave-one-out experimental analysis, and show that the outputs of the CNN (trained exclusively on other experimental datasets) faithfully replicate the well-known modulation of the P300 signal observed in the test set. The sources of P300 variability we examine include (1) perceptual similarity, (2) target-to-target interval, and (3) dual-task demands. We then show that the improved SNR in the CNN output space allows us to obtain the same level of significance when comparing experimental conditions using EEG derived P300 amplitude measures, while requiring substantially fewer trials. Finally, we examine a free-viewing target detection task, in which the anticipated neural response is masked by both subsequent eye movements as well as task-relevant visual feedback. Using our $\mathrm{CNN}$ approach, the underlying target-related neural response can be recovered, whereas with conventional P300 amplitude measurement techniques, it cannot.

\section{REVIEW OF THE P300 EVOKED RESPONSE}

The P300 evoked response, first reported by Sutton et al. (1965), is a stereotyped neural response to novel or task-relevant stimuli with maximal amplitude in the parietal region (Polich, 2007). In a two-stimulus paradigm, the P300 is most often observed as a response to an infrequent target stimulus presented among more frequently occurring background stimuli with typically 1-3 $s$ between stimulus presentations. In the three-stimulus version of the task, an additional infrequent "non-target" stimulus is presented along with the target and background. In either case, only the target stimulus requires a response from the observer, though overt responses (e.g., button press) are not required to elicit the P300. When visual stimuli are used and the rate of stimulus presentations increases to $2 \mathrm{~Hz}$ or greater, the approach is commonly referred to as rapid serial visual presentation (RSVP) (Chun and Potter, 1995).

It is well-established that both the amplitude and latency of the P300 are affected by endogenous, exogenous, and pathological factors (Jeon and Polich, 2003; Polich and Comerchero, 2003; Polich, 2007). This includes both feature (e.g., target and background similarity) and temporal (e.g., target frequency) stimulus properties, as well as cognitive states (e.g., high working memory load). As such, it provides an ideal event-related neural response from which to assess the effectiveness of our generalized neural decoding methodology. Prior research shows the P300 amplitude is generally larger with an earlier latency for tasks with easy vs. difficult target/background discrimination, in tasks with long vs. short target-to-target intervals, and in tasks with low vs. high task demands. Here we use P300 to indicate target-detection related activity showing maximum positive voltage over parietal electrodes. This is also referred to as the P3b, as opposed to the P3a elicited over more anterior electrodes by novel distractors (Polich, 2007).

\subsection{Perceptual Similarity}

Both the amplitude and latency of the P300 are modulated by the perceptual similarity between targets and non-targets. Specifically, P300 amplitudes in response to non-target stimuli tend to increase the more similar these stimuli are to target stimuli (Verleger, 1997; Azizian et al., 2006; Marathe et al., 2015; McDaniel et al., 2018). Variability in P300 responses evoked during an RSVP task can be attributed, in part, to the presence of distractor images that share physical and/or semantic characteristics with the rare target class of interest, interspersed with frequent background images (Polich and Comerchero, 2003). Studies have shown that when the target/background discrimination difficulty increases, that is, when the target is similar to the standard background, P300 amplitude decreases and latency lengthens (McCarthy and Donchin, 1981; Comerchero and Polich, 1999).

\subsection{Target-To-Target Interval}

Prior work has also shown that P300 amplitude can change as a function of the target stimulus probability, the number of background images preceding the target (i.e., target-to-target interval or TTI), as well as the interstimulus interval (ISI) (Gonsalvez and Polich, 2002). Targets at short ISIs tend to have smaller and longer latency P300 responses compared to those elicited at longer ISIs (Woods et al., 1980; Gonsalvez et al., 1999). However, when ISI is manipulated together with TTI, more of 
the P300 amplitude variance is accounted for by TTI than ISI (Gonsalvez and Polich, 2002). P300 amplitude remains relatively constant when TTIs approach 6-8 s or longer (Gonsalvez and Polich, 2002). Together these findings suggest that the neural system needs time to efficiently recover from processing targetrelated information.

\subsection{Task Demands}

Amplitude and latency effects have also been observed with changes in cognitive workload (Wickens et al., 1983; Handy et al., 2001; Pratt et al., 2011). Specifically, it has been shown that working memory manipulations from one task can affect multiple levels of neural processing in another. For example, in a study by Pratt et al. (2011), subjects performed an arrow flanker task either alone or while performing a Sternberg task with high or low working memory load. The results showed decreased P300 amplitude with increased working memory load for the incongruent flanker stimuli. This result has also been found in more applied settings as evidenced by decreased P300 amplitudes under high compared to low workload/baseline in an aircraft control task (Causse et al., 2016; Giraudet et al., 2016). P300 latency is also affected by task demands, as demonstrated in a recent study by Ries et al. (2016), which showed that target response latency significantly increased as a function of auditory working memory load. For a review on utilizing the P300 as an index of task difficulty see Kok (2001).

\section{MATERIALS AND METHODS}

\subsection{P300 Database}

Our P300 EEG database was constructed from four previously collected and analyzed P300 experimental datasets. All experiments were approved by the Institutional Review Board of the Army Research Laboratory. A high-level summary is given in Table 1. The database contains examples from both RSVP and free-viewing tasks, and were selected to investigate the decoding properties of our CNN approach. For our leave-oneout tests, by training a model using the three remaining datasets, we expected to sufficiently represent the variability in our hold-out set. An overview of the four experimental paradigms is shown in Figure 1.

TABLE 1 | Sources of variability, \# of Subjects, and \# of Trials for each P300 Dataset (T, Target; D, Distractor; B, Background).

\begin{tabular}{|c|c|c|c|c|c|}
\hline Dataset & $\begin{array}{l}\text { Perceptual } \\
\text { similarity }\end{array}$ & $\begin{array}{l}\text { Target } \\
\text { interval }\end{array}$ & $\begin{array}{c}\text { Task } \\
\text { demands }\end{array}$ & \# Subjects & $\begin{array}{c}\text { \# Trials } \\
(T+D, B)\end{array}$ \\
\hline 1 & $x$ & & & 18 & $\begin{array}{c}10,512- \\
99,504\end{array}$ \\
\hline 2 & $x$ & $x$ & & 17 & $\begin{array}{r}12,255- \\
275,494\end{array}$ \\
\hline 3 & & & $x$ & 16 & $\begin{array}{c}1,764- \\
17,649\end{array}$ \\
\hline 4 & $x$ & & $x$ & 16 & $\begin{array}{c}3,394- \\
31,506\end{array}$ \\
\hline
\end{tabular}

\subsubsection{Dataset 1: Target Discrimination With Distractor Images}

Our first dataset (Figure 1A) involved a $2 \mathrm{~Hz}$ RSVP task with targets, distractors, and background images (Marathe et al., 2015). Target to background ratio, and distractor to background ratio, were both approximately 1:14. Target images contained people holding a weapon, naturally positioned in a simulated urban environment, while distractor images contained people not holding weapons. Background images contained neither people nor weapons. To avoid interference from the attentional blink phenomena (Raymond et al., 1992), at least two background images were required to follow any target or distractor image. Each subject experienced a total of four conditions for this dataset, which were the combinations of two manipulations: (1) subjects either mentally counted or pressed a button in response to targets, and (2) the run either had no distractors (only target and background images), or had all image types (target, background, and distractor images). In all conditions, the target as well as distractor stimuli could be stationary or moving. The appearance of moving stimuli was created by presenting five sequential images within the $0.5 \mathrm{~s}$ stimulus epoch. In each image the target was in a slightly different location (i.e., animation). Data were collected from 18 subjects (13 male). EEG data were recorded with 64-channel BioSemi ActiveTwo (Amsterdam, Netherlands).

\subsubsection{Dataset 2: Target Discrimination With Complex Imagery and Variable Target-To-Target Intervals}

Our second dataset (Figure 1B) is from a $5 \mathrm{~Hz}$ RSVP task that incorporated variations in TTI as well as stimulus complexity (Touryan et al., 2014a,b). Subjects performed six 10-min blocks of target detection in which images of everyday office scenes were presented. There were five unique target categories: stairs, containers, posters, chairs, and doors. Before each block, the subject was notified of the target category for that block, and was instructed to push a button when a target stimulus was presented (i.e., go / no-go). There were six distinct experimental conditions where the TTI was varied. These six block types had average TTIs of 1.8, 2.2, 2.8, 3.9, 6.5, and 17.4 s. Targets also appeared at various sizes, eccentricities, and occlusion levels (i.e., target object could be fully visible or occluded by other objects in the scene), increasing the complexity of image categorization. Due to the difficulty of this task (on average, $44 \%$ of targets were missed), only trials with correct responses were used for this analysis. Data were collected from 17 subjects ( 7 male). EEG data were recorded with 256-channel BioSemi ActiveTwo. Channels were downsampled to the 64 BioSemi system montage.

\subsubsection{Dataset 3: Target Discrimination During Guided Visual Search Under Varying Degrees of Cognitive Workload}

The third dataset (Figure 1C) involved guided fixations around a grid of target and non-target stimuli, in both the presence and absence of an auditory task (Ries et al., 2016). Eye movements were guided through the use of a red annulus across a grid of mostly L's. The annulus moved to a new location approximately every second, and subjects made a button press when they fixated 


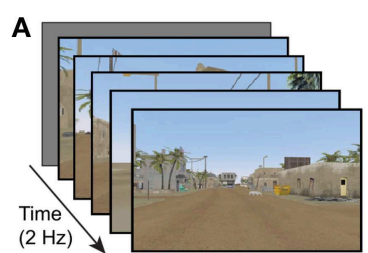

B

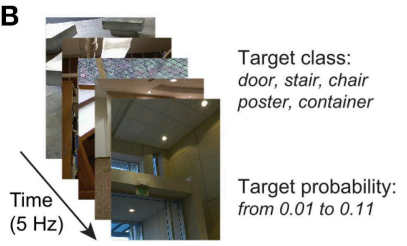

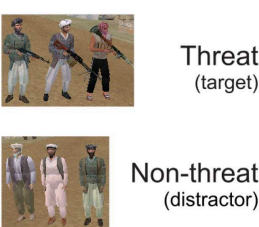

Easy (correct) targets: door

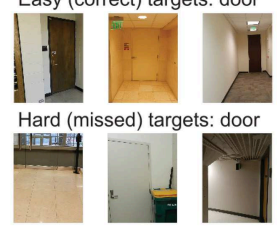

C

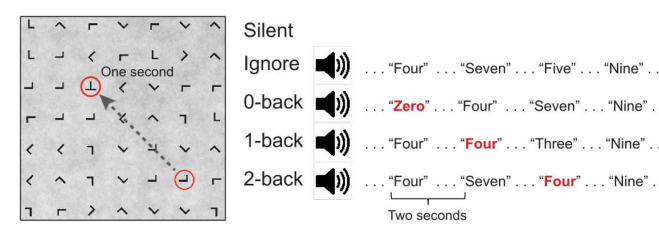

D
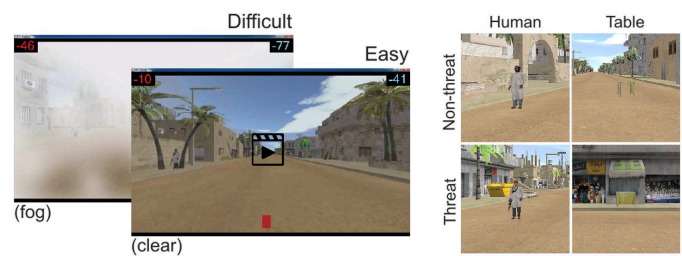

FIGURE 1 | Experimental paradigms from the four datasets (A-D) used in this analysis.

on the infrequent target letter, T. The guided visual search task was performed under five conditions: (1) alone (Silent), (2) while ignoring binaurally presented digits, numbers 0-9 (Ignore), or (3-5) while using the auditory digits in a 0,1 , or 2-back working memory task (0-, 1-, 2-Back). Auditory stimuli were presented every $2 \mathrm{~s}$ with a $500 \mathrm{~ms}$ offset from the red annulus to prevent simultaneous auditory/visual events. Data were collected from 16 subjects (all male). Eye tracking data were acquired using a SMI RED 250 (Teltow, Germany). EEG data were recorded with a 64-channel BioSemi ActiveTwo. Horizontal and vertical electrooculogram (EOG) data were recorded, respectively, by placing electrodes near the outer canthus of each eye, and above and below the orbital fossa of the right eye.

\subsubsection{Dataset 4: Target Discrimination via Free-Viewing Visual Search During Video Playback}

The fourth dataset (Figure 1D) is from a free-viewing task in which subjects viewed an urban landscape in a 15-min video (Passaro et al., 2017; Solon et al., 2018). In this video, subjects were driven through a simulated urban environment and required to find two different types of targets, humans and tables, and then discriminate between visually similar versions of each entity. Human entities were either holding a weapon (threat) or unarmed (non-threat). Tables were oriented in such a way that they could either hide an explosive device (threat) or not (non-threat). Target stimuli would abruptly appear one at a time in random but logical locations (i.e., on the street or in a doorway) at an approximate rate of once every $3 \mathrm{~s}$, remaining on screen for $1 \mathrm{~s}$. Subjects were free to scan the environment but were instructed to indicate the type of entity (threat or non-threat) by pressing a button with either the left or right index finger (i.e., two-alternative forced choice). Therefore, while the task was free-viewing, accurate discrimination tended to require that the subject fixate on the object. Participants were not required to hold target fixations for a prescribed amount of time, and thus subsequent fixations, along with other contaminating EOG effects, could occur once the subject had acquired enough information about the target to perform discrimination.
Subjects performed this task under two distinct visibility conditions: (1) a baseline of clear visibility (Clear) for easy stimulus detection, and (2) low visibility with an obscuring fog $(F \circ g)$ for difficult stimulus detection. Subject responses were graded for speed and accuracy, and the subject received performance feedback for every target occurrence. The per trial score was visually displayed on the screen after button press response. Following the offset of each target stimulus, a cumulative score bar was updated, and displayed at the top and bottom of the screen. Data were collected from 16 subjects (all male). EEG data were recorded with a 64-channel BioSemi ActiveTwo. Horizontal and vertical EOG data were recorded in the same fashion as Dataset 3.

\subsection{CNNs for Neural Decoding}

CNNs for image processing typically require vast amount of training data, often on the order of millions of images distributed across thousands of classes (Deng et al., 2009; Xiao et al., 2010; Zhou et al., 2018). In neuroimaging studies, however, collecting such vast amounts of data is prohibitively expensive and impractical, thus necessitating the need for $\mathrm{CNN}$ architectures designed specifically for low-sample neuroimaging data, while still being robust to both inter- and intra- subject differences. Early work applying CNNs to EEG signal classification was done by Cecotti and Graser (2011) for classification of subjectspecific P300 responses and by Ren and Wu (2014) and Sakhavi et al. (2015) for classification of imagined movements. Recent work by Lawhern et al. (2018) has yielded a CNN architecture for EEG (EEGNet) that can learn from relatively small amounts of data, on the order of hundreds of trials per subject. Furthermore, (Lawhern et al., 2018; Waytowich et al., 2018) showed that EEGNet enabled cross-subject transfer performance equal to or better than conventional approaches for several EEG classification paradigms, both event-related and oscillatory. EEGNet is also the model used to obtain our crossexperiment results described in Gordon et al. (2017), McDaniel et al. (2018), and Solon et al. (2018).

EEGNet is a compact CNN that takes minimally processed time-series EEG data as input, first using temporal convolutions 
that act as bandpass frequency filters, followed by depthwise spatial convolutions that act as spatial filters, which together improve SNR and reduce the dimensionality of the data. The depthwise convolution allows the model to learn spatial filters for each temporal filter, without being fully connected to all of the outputs of the previous layer and thus greatly reduces the number of parameters to be learned. EEGNet uses separable convolutions to more efficiently combine information across filters (Chollet, 2015). Each convolution layer is followed by batch normalization (Ioffe and Szegedy, 2015), 2D average pooling, and Dropout layers (Srivastava et al., 2014). We fit the EEGNet-4,2 model, denoting four temporal filters of length 64 samples and two spatial filters per temporal filter, as described in Lawhern et al. (2018) as it was previously shown to perform well for crosssubject P300 classification when compared to existing CNNbased models. In addition, it is a smaller model (measured by the number of free parameters) compared to existing models, making it faster to train.

\subsection{EEG Signal Preprocessing}

For all EEG datasets analyzed in this paper, the data were bandpass filtered between 0.3 and $50 \mathrm{~Hz}$ before being downsampled to $128 \mathrm{~Hz}$. To ensure that all datasets were scaled similarly, we normalized each subject in each experiment by dividing the filtered data by its median absolute deviation (MAD), calculated over all channels and time points. We use MAD to reduce the effect of transient but large artifacts that occurred in some of the EEG recordings.

\subsection{Model Training}

All CNN models were trained using a leave-one-experimentout procedure, combining data across all but one experiment for training and then testing on the held-out dataset. We used EEG epochs 1s in length for our training instances. For all datasets, with the exception of Dataset 4 , instances were created by epoching $[0,1] \mathrm{s}$ around stimulus or fixation onset. In Dataset 4, the P300 responses appear to begin prior to fixation, and therefore training instances were epoched [ $-0.30 .7] \mathrm{s}$ around fixation onset (Solon et al., 2018).

Models were trained to perform a binary classification between instances that contained a P300 response [response to target ( $\mathrm{T}$ ) and distractor (D) stimuli], and those that did not [responses to background stimuli (B)]. When present, distractors were included in the target class to capture the natural variability of the P300 response, as they elicit attenuated P300 responses (Azizian et al., 2006). To handle imbalance in both class (more non-targets than targets) and experiment size (some experiments had more instances than others), we applied a sample weighting procedure during training to ensure each class and experiment contributed equally. This was done using the inverse proportion of the data in the training dataset. For example, if the target to background ratio was 1:4, the sample weight for all target trials was set to 4 , while the sample weight for all background trials was set to 1 . The weights to control for experiment imbalance were calculated in a similar manner. The weights to control for class imbalance and experiment imbalance were multiplied together to form the final sample weights used in model training. The EEGNet-4,2 model was implemented in Tensorflow (Abadi et al., 2015), using the Keras API (Chollet, 2015). The model was trained for 100 iterations using the Adam optimizer with default parameter settings (Kingma and Ba, 2014), with a minibatch size of 64 instances, optimizing a categorical cross-entropy loss function. We trained this model for 100 iterations as we anecdotally observed minimal change in the training set cross-entropy loss going beyond 100 iterations. The dropout probability was set to 0.25 for all layers. Source code for the models can be found at https://github.com/vlawhern/ arl-eegmodels. We will refer to the EEGNet-4,2 model as the "CNN Model," and its model outputs as "CNN Outputs," for the remainder of the paper.

\subsection{Model Testing}

Once the model was trained, we applied it to the test set using the sliding window approach depicted in Figure 2. For all datasets, we made a prediction (using $1 \mathrm{~s}$ of EEG as input) every six samples over a duration beginning at $1 \mathrm{~s}$ before, and ending at $1 \mathrm{~s}$ after, stimulus or fixation onset. For example, a CNN output at $\mathrm{T}$ $=0$ uses the EEG epoch $[0,1] \mathrm{s}$ as input, and a CNN output at $\mathrm{T}=$ 0.5 uses the EEG epoch $[0.5,1.5] \mathrm{s}$ as input. This produced a timeseries of $65 \mathrm{CNN}$ outputs, with each CNN output summarizing $1 \mathrm{~s}$ of EEG data. Each output can be considered a probability that the $1 \mathrm{~s}$ long input epoch of EEG data contained the same neural response associated with target stimuli extracted from the training data. It is important to note that, depending on the step size, neighboring points may be highly correlated. For example, a step size of $100 \mathrm{~ms}$ would produce neighboring points that were computed using data with $90 \%$ overlap. As a result, sharp deviations in the original signal, such as the relatively instantaneous phase resetting that initiates the P300 response, are difficult to pinpoint in time using our CNN outputs. Our modeling approach is more suited for assessing amplitude shifts that result from changes in the underlying signal as well as large latency shifts in the evoked response. Minor variances in latency, such as temporal jitter in the component waveforms, should be largely ignored by our convolutional approach. We show these sliding window outputs to provide a more complete perspective of model performance.

\subsection{Statistical Testing for Signal to Noise Ratio Analysis}

For our SNR analysis, using Dataset 3, we performed pairwise statistical testing on both P300 amplitudes as well as CNN outputs. The steps that we followed for testing P300 amplitudes were to (1) compute the average over a parietal region of interest (ROI) using channels Pz, P1, P2, CP1, CP2, and CPz in the 10-20 montage, (2) identify the mean value, per trial, in the window 400-850 ms post-stimulus or fixation onset, (3) remove, per subject, the mean of (2) for background trials from the means computed for target trials, (4) aggregate the data across subjects, and then (5) compare the distribution of target means in the Silent condition to target means in the 2-Back condition using a 1-tailed $T$-test. The steps that we followed to compare CNN outputs were similar to those used for P300 amplitudes, with the exception that we used the $\mathrm{CNN}$ output at $T=0 \mathrm{~s}$ rather 


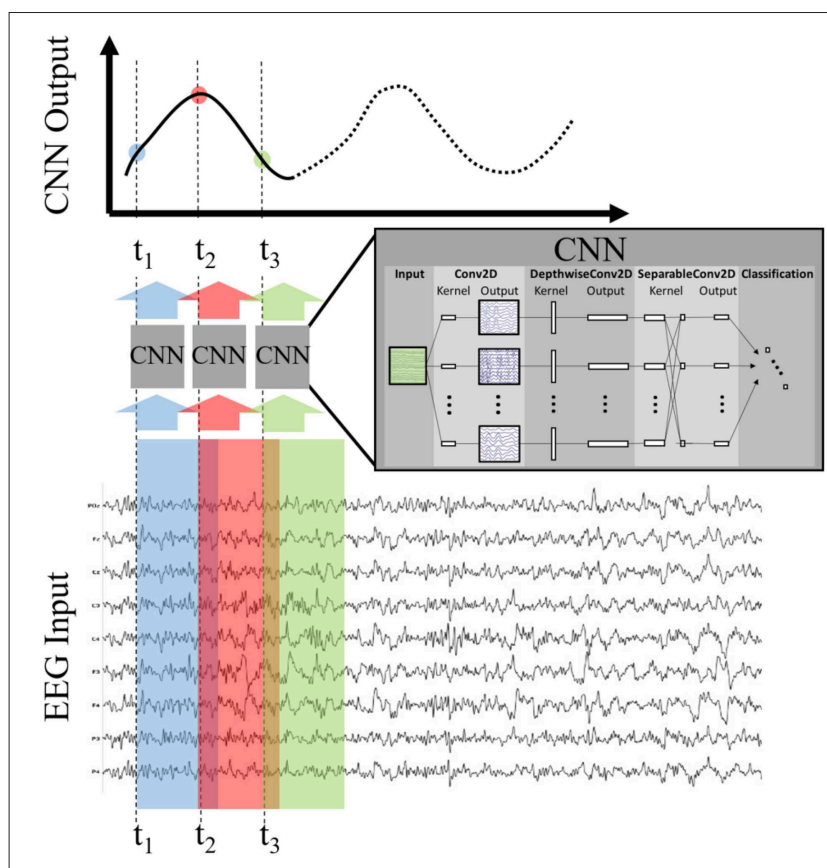

FIGURE 2 | Production of CNN Outputs. Each CNN output is produced by passing as input an epoched sample of the EEG, i.e., a multi-dimensional array of channels $\times$ time. By stepping the CNN over the EEG record our approach produces an output signal that is decoded, in time and space, by the encoded deep model. For convenience purposes, we record the CNN output times using the first time point of the EEG epoch in question.

than means computed over time and space. In other words, we computed the average non-target CNN output, per subject, and subtracted that value from the per trial response for targets. We aggregated the target values across subjects and performed a $T$ test to compare the distributions of CNN outputs for targets in the Silent condition to targets in the 2-Back condition.

In section 4.4 we report the resulting $\mathrm{p}$-values for Dataset 3 as a function of the percentage of trials selected. Trials were randomly downselected per subject, and only selected trials were used to perform both the P300 amplitude and CNN output tests. To reduce noise in our comparison resulting from trial selection, we repeated our random sampling and testing procedure 100 times for each percentage value analyzed.

\section{RESULTS}

\subsection{Perceptual Similarity}

Figure 3A shows the P300 response, along with standard errors, as a difference wave for the target and distractor stimuli (i.e., target minus background, distractor minus background) for Dataset 1, aggregated across subjects. For this test, we compute our Evoked Responses and CNN outputs only for the condition that employs mental counting only (i.e., no button press) and has both target and distractor stimuli. The data for these plots was computed from the average over a parietal ROI (channels $\mathrm{Pz}$, $\mathrm{P} 1, \mathrm{P} 2, \mathrm{CP} 1, \mathrm{CP} 2$, and $\mathrm{CPz}$ ) and utilized the same preprocessing used to prepare the data for the CNN model. The CNN model was trained on Datasets 2, 3, and 4. Figure 3B shows the average
CNN outputs for the same conditions presented in Figure 3A. We see that the model outputs closely mirror the P300 amplitude differences; in both plots, a clear separation of amplitudes is visible between target and distractor responses. In Figure $\mathbf{3 B}$ the CNN outputs peak at $\mathrm{T}=0$, which is consistent with the model training, given that both use epochs $[0,1]$ s around stimulus onset.

\subsection{Target-To-Target Interval}

Figure 4A shows the P300 response as a function of TTI for Dataset 2. Shorter TTIs (i.e., more frequent target presentations) measurably attenuate P300 amplitude. As TTI increases, the amplitude of the P300 response also increases. Figure 4B shows the model outputs for the same conditions presented in Figure 4A. It should be noted that the responses in Figure 3A appear faster, yet persist longer, than the responses in Figure 4A. The baseline period, -0.5 to $0 \mathrm{~s}$, in Figure 3A shows greater desynchronization (i.e., negative dip). Although the origins of these differences are not critically important for our current analysis, it is important that the cross-experiment-trained models represented the amplitude shifts with a similar degree of fidelity, while also reflecting the subtle temporal shifts and overall shorter responses in Figure 4A. The CNN outputs in Figure 4B are shifted to the right and the main lobe appears thinner.

\subsection{Task Demands}

We compare the P300 waveforms from Dataset 3, in which subjects performed a guided visual search task while simultaneously performing an auditory N-back task at varying degrees of difficulty. Figure 5 presents the results for conditions Silent, 0-Back, and 2-Back for both the P300 response (A) and model outputs (B). The high workload P300 waveforms have, on average, lower peak amplitudes and longer latencies. The model outputs reflect both of these shifts, though the shift in amplitude is much stronger than the shift in latency. That the model outputs continue to reflect shifts in amplitude and latency for fixation-locked P300 responses, when the majority of the training data were stimulus-locked, is further indication that the underlying template encoded within the CNN is based on a generalized representation of the P300 response. This template is reasonably invariant to the other experimentally-contingent components or induced artifacts, whether those are visualevoked responses from rapid image presentations, or saccadic spikes/EOG contamination during visual search.

\subsection{Signal to Noise Ratio Analysis}

We performed the following analyses to assess the extent to which CNN-based decoding improves SNR. In the first test, we systematically downselected the amount of data extracted from a test set, Dataset 3, to compare the outcome of statistical testing as a function of the amount of data available. For this test, we compared data from the Silent and 2-Back conditions; the results of this test are shown in Figure 6. As can be seen in the figure, the P300 amplitude analysis produces a p-value of approximately 0.01 using $100 \%$ of the data. An equivalent p-value can be obtained using the $\mathrm{CNN}$ output with roughly $25 \%$ of the data. Of course, the decision to reject the Null Hypothesis (i.e., that target amplitudes are the same in both conditions) is a function 

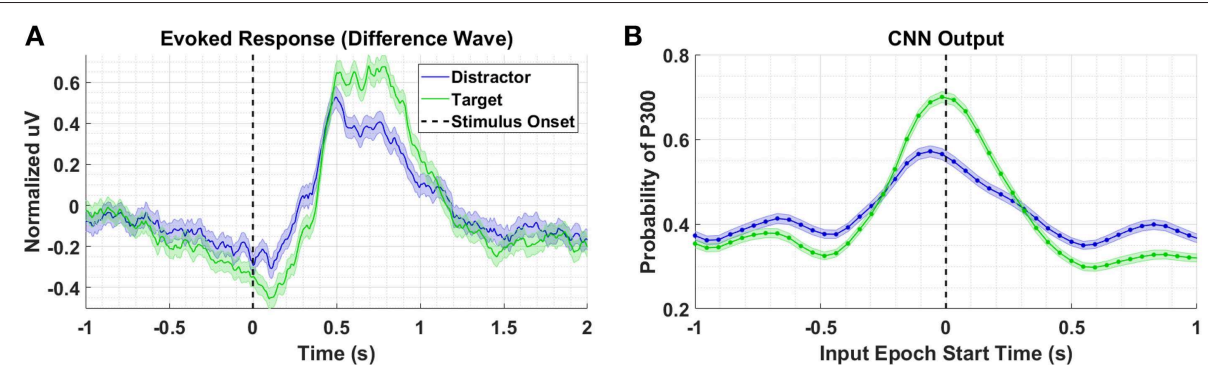

FIGURE 3 | Perceptual Similarity Results using Dataset 1. (A) Averaged P300 Evoked Response plots for target and distractors computed as difference waves for all subjects measured at the parietal ROI. (B) CNN outputs are generated, for the same trials shown in (A), via sliding window approach detailed in Figure 2. Starting at 1s before stimulus onset, CNN outputs are generated (using $1 \mathrm{~s}$ epochs of EEG data) every 6 samples, resulting in a time-series of 65 total predictions. Shaded regions in both figures denote 2 standard errors of the mean.
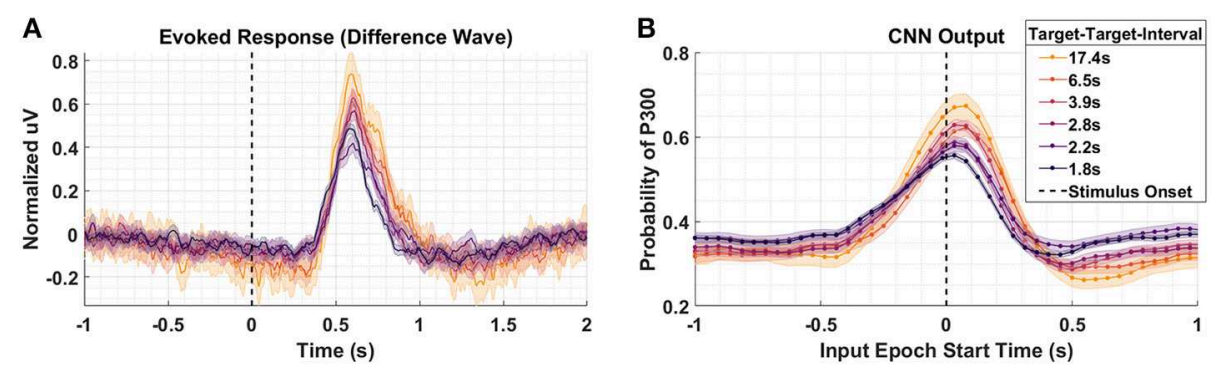

FIGURE 4 | Target-to-Target Interval using Dataset 2. (A) Averaged P300 Evoked Response (computed as difference waves) measured at the parietal ROI across subjects for the 6 distinct experimental conditions with varied TTI. The 6 conditions have, on average, TTI values of 1.8, 2.2, 2.8, 3.9, 6.5, and 17.4 s. (B) A time-series of 65 CNN outputs are generated, for the same Target trials shown in (A), via sliding window approach detailed in Figure 2. Shaded regions in both figures denote 2 standard errors of the mean.
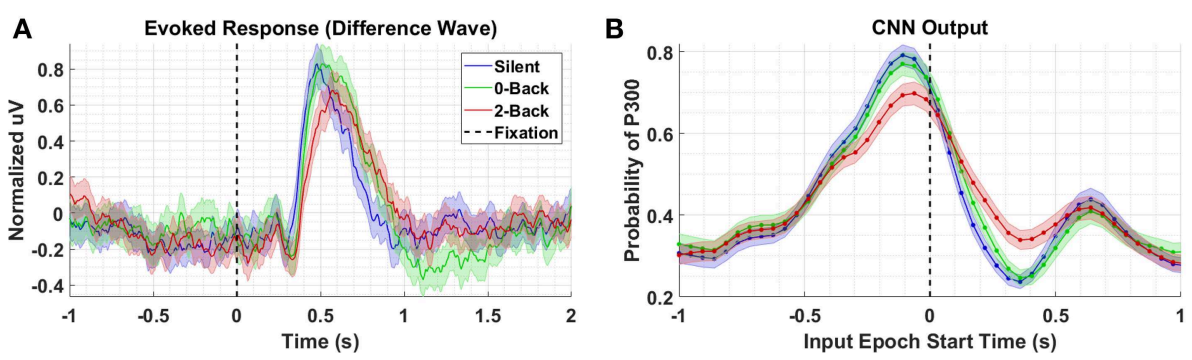

FIGURE 5 | Task Demands results using Dataset 3. (A) Averaged P300 Evoked Response (computed as difference waves) for targets trials, time-locked to fixation onset, obtained from the parietal ROI for the Silent, 0-Back, and 2-Back conditions (B). A time series of 65 CNN outputs are generated, for the same trials shown in (A), via sliding window approach detailed in Figure 2. Shaded regions in both figures denote 2 standard errors of the mean.

of several factors, including significance threshold and multiplecomparison corrections. In the original paper (Ries et al., 2016), the authors did not report a significant difference in amplitudes after performing a multiple-comparisons correction. Use of CNN outputs would have affected this result due to the enhanced SNR of the CNN method.

To better understand what is happening in the temporal domain, we performed a follow-up analysis on Dataset 2 . We artificially created three groups of data from this dataset by grouping correct target responses by reaction time. The groups we considered were fast, medium, and slow RTs, which were calculated using the 33rd and 66th percentiles as cutoffs. These results are presented in Figure 7 and Table 2. As can be seen from Figure 7A, the P300 response for fast and medium responses appear relatively similar in amplitude, but the medium response is temporally shifted $\sim 100 \mathrm{~ms}$ to the right of the fast response, which is consistent with the measured reaction times presented in Table 2. The P300 response for the slow group is shifted $\sim 100$ $\mathrm{ms}$ to the right of the medium response; however, the amplitude appears severely attenuated, while the lobe width appears wider. This phenomenon is largely a result of the unequal variance in the slow RT group; the P300 response in these trials is not 

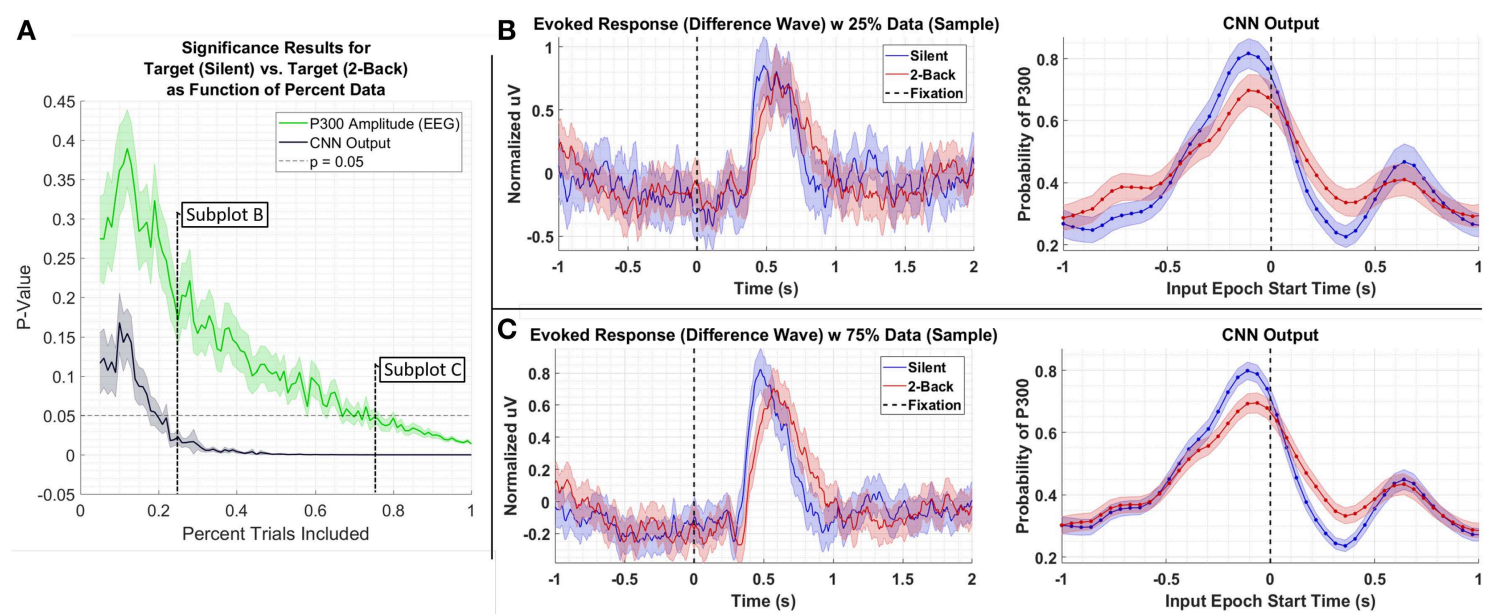

FIGURE 6 | (A) Result of significance testing for amplitude differences between target response in the Silent vs. 2-Back conditions in Dataset 3 using CNN outputs (black) and measured P300 amplitude (green). (B) Sample of the P300 and CNN outputs using 25\% of the data. (C) Sample of the P300 and CNN outputs using $75 \%$ of the data.

necessarily diminished, but rather the increase in RT variance diminishes the amplitude of the averaged response (Verleger et al., 2005). Inspecting the results in Figure 7B, we observe that the time course of $\mathrm{CNN}$ outputs reflect the overall temporal trend of the three groups, i.e., the peak of the fast response is $\sim 100 \mathrm{~ms}$ before the peak of the medium response, which is itself about $\sim 100 \mathrm{~ms}$ before the peak of the slow response. In other words, a temporal shift of the entire distribution of the P300 response also shifts the CNN output peaks. Unlike the raw amplitude measurements, though, the CNN outputs indicate no difference in amplitude between the fast and slow responses. The convolutional structure of the $\mathrm{CNN}$ has, effectively, resolved the increased temporal variability in the slow group. There does appear to be a difference in amplitudes between the medium response and the response of the fast/slow groups; however, this can be explained by remembering that the CNN outputs are probability assessments that the input signal matches a pretrained template, which is maximized in this case by the medium group. The shifts in amplitude as a function of latency are distributed about the center axis, i.e., $\mathrm{T}=0 \mathrm{~s}$, in Figure 7 .

Returning to Figures 6B,C, we see that there is a minor, observable, shift in latency, which could impact the amplitude measurements of our CNN. However, the shift in latency for the low amplitude group (i.e., 2-Back) is toward the center axis $(\mathrm{T}=0 \mathrm{~s})$, where one would expect the CNN probability estimates to peak. In other words, the result in Figure 7 would suggest that if the amplitudes in Dataset 3 were equal, we would observe a lower amplitude for the Silent condition than the 2Back condition, due to the leftward shift of the Silent response. We argue that by observing the opposite trend, our confidence in the result increases.

\subsection{Application to Complex Data}

In our previous work (Solon et al., 2018) utilizing Dataset 4, we show that in the Clear condition, the fixation-locked and stimulus-locked neural responses are largely identical, with the exception of a small time shift. Here, the target's "pop-up" effect acts an exogenous, or peripheral, cue that reliably guides the subject's attention to the target. When the scene is obscured by fog, the latencies between stimulus onset and its subsequent fixation become longer and more variable, as the effect of this peripheral cue is diminished, and in some cases eliminated. As a result, subjects must employ more top-down search strategies to discover the target, which they may only perceive once it is within their parafoveal vision. Consequently, we expect that in the Clear condition, that the P300 response will be primarily locked to stimulus onset, whereas in the Fog condition, that the P300 responses are mixed between stimulus onset locking and fixation onset locking.

The latency between stimulus and a peripherally cued fixation is relatively stereotyped, with the most common fixation onset occurring $0.2194 \mathrm{~s}$ post stimulus. We arrived at this value by fitting a lognormal probability distribution to a histogram of target fixation onset times in the Clear condition only, and choosing the peak value of the fitted distribution (the distribution of fixation onsets was heavily right skewed). In the Fog condition, we expect fixations that occur faster than this value to have predominantly fixation-locked neural responses, as these fixations occur faster than they would have if they were peripherally cued. In other words, considering the standard stimulus-to-fixation timing in the easier Clear condition, the subjects would not have had time to receive the peripheral cue and then saccade to the object. In Figure 8, we compare four conditions: Fog and Clear with short fixation onset times, and Fog and Clear with long fixation onset times. The Fog with short fixation times represents the case where the subject relies on no peripheral cuing. Short fixation onset times are defined as being less than $0.2194 \mathrm{~s}$, whereas long fixation onset times are defined as being greater than or equal to $0.2194 \mathrm{~s}$. 

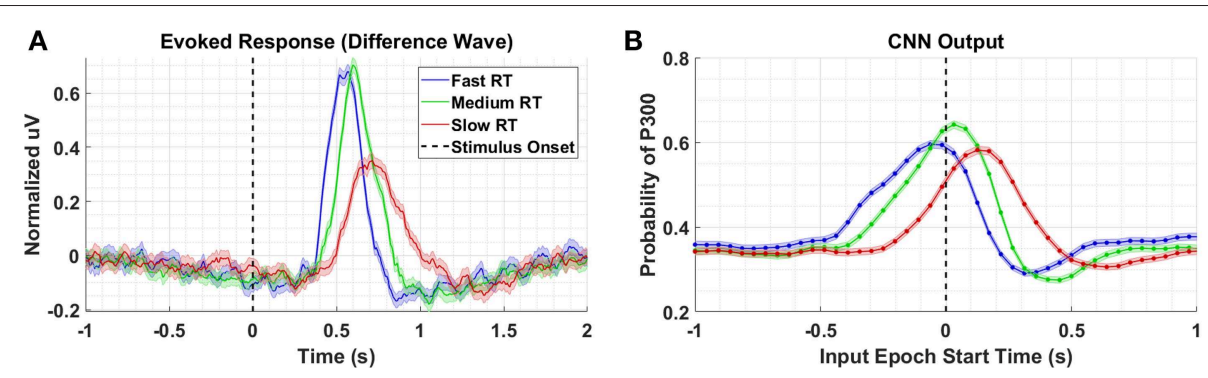

FIGURE 7 | (A) Averaged P300 Evoked Response (computed as difference waves) for correct target trials for each RT bin, detailed in Table 2 (B). A time series of 65 CNN outputs are generated, for the same trials shown in (A), via sliding window approach detailed in Figure $\mathbf{2}$. Shaded regions in both figures denote 2 standard errors of the mean.

TABLE 2 | Mean and Standard Deviation values for Fast, Medium, and Slow reaction time groups.

\begin{tabular}{lcc}
\hline Category & Average reaction time (s) & STD of reaction time (s) \\
\hline Fast & 0.428 & 0.051 \\
Medium & 0.540 & 0.031 \\
Slow & 0.740 & 0.135 \\
\hline
\end{tabular}

In the Evoked Response difference waves shown in Figure 8A, the differences between the conditions are imperceptible. Importantly, the Evoked Response wave forms are atypical, with the apparent response persisting well after $1 \mathrm{~s}$ post fixation, a result, we believe, of the mixture of subsequent search fixations (which occur $\sim 0.65 \mathrm{~s}$ after target fixation) and visual feedback indicating the subject's game score $(\sim 0.5 \mathrm{~s}$ after target fixation). In Figure 8B, we see that the $\mathrm{CNN}$ outputs for the Clear condition, both slow and fast, are similar to each other in timing and magnitude despite the difference in fixation latency relative to stimulus onset. However, the neural response, as measured by the CNN output, associated with short fixations (red-763 trials) occurs closer to fixation onset than the neural responses associated with long fixations (black- 1,676 trials). For the Fog condition, we see that the CNN outputs associated short fixations (blue-101 trials) peak at $\mathrm{T}=0 \mathrm{~s}$, which confirms our hypothesis that the neural responses are driven by top-down search rather than peripheral cuing. In other words, processing of these stimuli begin at fixation. For long fixation onsets in the Fog condition (green-854 trials) we see two peaks in the CNN output: one that begins prior to fixation onset, and one that begins at fixation onset. We hypothesize that the first of these peaks represents the Fog targets, despite their obfuscation, that subjects were able to detect through peripheral cuing. The second represents the trials with slow-fixation-onset where peripheral cuing did not play a significant role in target discovery.

\section{DISCUSSION}

Existing methods to analyze neural phenomena often require the collection of large numbers of precisely-timed trials due to the relatively poor SNR of minimally processed EEG data. Here we introduced a technique that utilizes convolutional deep-networks to decode EEG data. We applied this neural decoding approach to the analysis of the well-known P300 response. The P300 response has previously been shown to vary in both amplitude and latency as a result of a variety of experimental manipulations. We showed that the outputs of our CNN were sensitive to these changes, whether those changes occurred as a result of perceptual similarity, target interval, or task demands. We further showed that our CNN-based decoding approach improved overall SNR of the underlying EEG signal, allowing us to correctly reject the null hypothesis for a comparison of amplitude means using only a fraction of the recorded data. We also demonstrated that the CNN outputs shift with latency of the underlying signal, but that temporal variability about a shifted mean does not impact CNN output to the same extent that it impacts averaged amplitudes. Perhaps most importantly, our technique can be completely pretrained using previously run experiments. Taken together, these results validate the use of $\mathrm{CNN}$ architectures for generalized neural decoding and show that such decoding can substantially alleviate the need for large numbers of repeated trials.

In section 4.5 , we provide an example application where we utilized a pre-trained CNN model to decode P300 variability in the previously unseen Dataset 4 . In the future, this approach could be applied to similarly challenging datasets, especially those where few trials are available per experimental condition. Likewise, our CNN decoding method may demonstrate some utility as a feature extractor for brain computer interface (BCI) applications. For example, previous studies have used P300 amplitude measures as features for predicting cognitive workload (Brouwer et al., 2012). Using CNN outputs, as opposed to P300 amplitude features or linear classifiers, may provide more accurate estimates of workload without the burden of additional training or calibration data.

However, there are limitations to our CNN decoding approach. Principally, for our chosen neural response, the P300 evoked response, the $\mathrm{CNN}$ model loses temporal resolution. This is a direct consequence of the windowing and temporal convolutions within the model that are designed to reduce the effects of temporal jitter. Whether this resolution is truly lost or must be further decoded by analyzing the hidden layers and internal activity of the network is an important question that should be addressed with future work. Of 

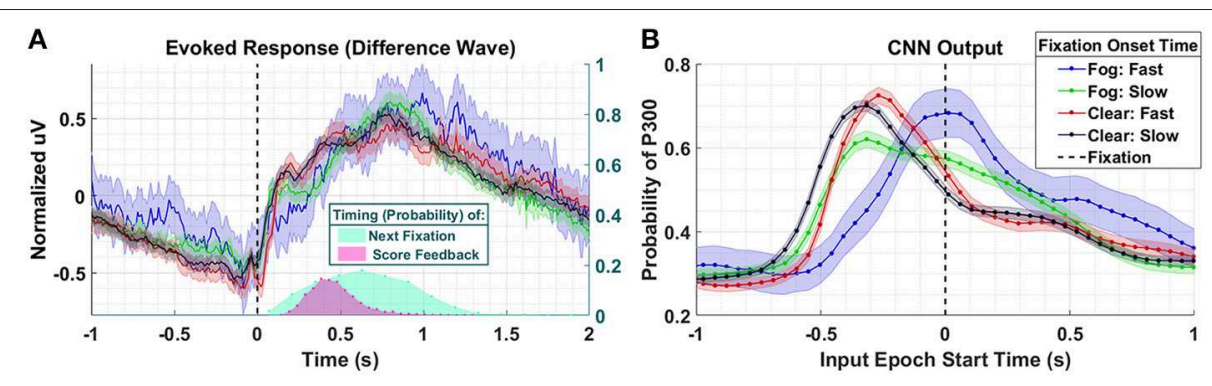

FIGURE 8 | Results using Dataset 4. (A) Averaged P300 Evoked Response (computed as difference waves) for target trials (left axis) time-locked to fixation onset, obtained from the parietal $\mathrm{ROI}$ and distribution of subsequent fixations as well as visual feedback time-locked to fixation onset (right axis). Fast fixation onset times represent those which occur faster than those which could have been peripherally cued (B). A time series of 65 CNN outputs are generated, for the same trials shown in (A), via sliding window approach detailed in Figure 2. Shaded regions in both figures denote 2 standard errors of the mean.

course, this also points at another potential drawback of the CNN-based approach, which is the overall interpretability of layered networks. Techniques for interpreting hidden layers and activity of deep networks have advanced greatly over the past few years, primarily in computer vision (Zeiler and Fergus, 2014; Shrikumar et al., 2017; Ancona et al., 2018; Montavon et al., 2018). These techniques have recently been applied to neurophysiological data like EEG (Sturm et al., 2016; Lawhern et al., 2018), enabling additional insights into CNN-derived feature representations. However, algorithms and approaches to fully enable the interpretation and understanding of network behavior remains an open scientific question. In addition, while CNN decoding improves amplitude measurements in the presence of temporal jitter, large temporal shifts can create false changes in amplitude as a result of the template matching occurring within the CNN (see Figure 7 fast vs. medium response). The relationship between latency and measured amplitude can be a confound in P300 analysis. If the purpose of neural decoding includes amplitude comparisons at different latencies, one possible solution would be to tailor the training data to the anticipated latencies of interest.

Finally, as our decoding approach (1) can be pre-trained, (2) is capable of ignoring co-morbid, experimentally induced components or artifacts, and (3) exhibits a certain amount of invariance to temporal uncertainty, this work represents an important extension beyond the existing approaches, data driven or otherwise, employed in the measurement and interpretation of neural phenomena. We believe the use of such CNN-based decoding will enable more complex, real-world neuroscience research (Blankertz et al., 2010; van Erp et al., 2012; Saproo et al., 2016). By allowing the experimenter to develop decoding models from precisely controlled laboratory experiments, yet analyze data from a much smaller number of trials without requiring the same level of temporal precision, CNNs such as the one presented here, can help bridge the gap between our knowledge of how the brain functions in the laboratory and how it may function in the real-world. Although there remains additional work to develop, refine, and validate CNN decoding approaches, including different neural phenomena and brain states, we anticipate a growing reliance on deep models for neuroscientific research in complex, realworld environments.

\section{DATA AVAILABILITY}

The datasets for this manuscript are available upon request. Requests to access the datasets should be directed to the corresponding author.

\section{ETHICS STATEMENT}

The voluntary, fully informed consent of the persons used in this research was obtained in written form. The document used to obtain informed consent was approved by the U.S. Army Research Laboratory's Institutional Review Board (IRB) in accordance with 32 CFR 219 and AR 70-25, and also in compliance with the Declaration of Helsinki. The study was reviewed and approved by the U.S. Army Research Laboratory's IRB before the study began.

\section{AUTHOR CONTRIBUTIONS}

AS and SG designed scientific tests and analyzed the data. AS, VL, and SG developed the convolutional neural network model and cross experiment training process. AS, SG, JT, VL, AR, and JM wrote the manuscript.

\section{FUNDING}

This project was sponsored by the US Army Research Laboratory under Cooperative Agreement Number W911NF-10-2-0022. The views and conclusions contained in this document are those of the authors and should not be interpreted as representing the official policies, either expressed or implied, of the US Government. The US Government is authorized to reproduce and distribute reprints for Government purposes notwithstanding any copyright notation herein. 


\section{REFERENCES}

Abadi, M., Agarwal, A., Barham, P., Brevdo, E., Chen, Z., Citro, C., et al. (2015). TensorFlow: Large-Scale Machine Learning on Heterogeneous Systems. Available online at: https:tensorflow.org

Ancona, M., Ceolini, E., Öztireli, C., and Gross, M. (2018). "Towards better understanding of gradient-based attribution methods for deep neural networks," in International Conference on Learning Representations (Vancouver, BC).

Azizian, A., Freitas, A., Watson, T., and Squires, N. (2006). Electrophysiological correlates of categorization: P300 amplitude as index of target similarity. Biol. Psychol. 71, 278-288. doi: 10.1016/j.biopsycho.2005.05.002

Blankertz, B., Tangermann, M., Vidaurre, C., Fazli, S., Sannelli, C., Haufe, S., et al. (2010). The berlin brain-computer interface: non-medical uses of bci technology. Front. Neurosci. 4:198. doi: 10.3389/fnins.2010.00198

Brouwer, A.-M., Hogervorst, M. A., van Erp, J. B. F., Heffelaar, T., Zimmerman, P. H., and Oostenveld, R. (2012). Estimating workload using EEG spectral power and ERPs in the n-back task. J. Neural Eng. 9:045008. doi: 10.1088/1741-2560/9/4/045008

Causse, M., Peysakhovich, V., and Fabre, E. F. (2016). High working memory load impairs language processing during a simulated piloting task: an erp and pupillometry study. Front. Hum. Neurosci. 10:240. doi: 10.3389/fnhum.2016.00240

Cecotti, H., Eckstein, M. P., and Giesbrecht, B. (2014). Single-trial classification of event-related potentials in rapid serial visual presentation tasks using supervised spatial filtering. IEEE Trans. Neural Netw. Learn. Syst. 25, 20302042. doi: 10.1109/TNNLS.2014.2302898

Cecotti, H., and Graser, A. (2011). Convolutional neural networks for p300 detection with application to brain-computer interfaces. IEEE Trans. Pattern Anal. Mach. Intell. 33, 433-445. doi: 10.1109/TPAMI.2010.125

Chollet, F. (2015). Keras. Available online at: https://github.com/keras-team/keras (accessed March 6, 2019).

Chun, M. M., and Potter, M. C. (1995). A two-stage model for multiple target detection in rapid serial visual presentation. J. Exp. Psychol. Hum. Percept. Perform. 21:109. doi: 10.1037//0096-1523.21.1.109

Comerchero, M. D., and Polich, J. (1999). P3a and p3b from typical auditory and visual stimuli. Clin. Neurophysiol. 110, 24-30. doi: 10.1016/S0168-5597(98)00033-1

Deng, J., Dong, W., Socher, R., Li, L., Li, K., and Fei-Fei, L. (2009). "Imagenet: A large-scale hierarchical image database," in 2009 IEEE Conference on Computer Vision and Pattern Recognition (Miami Beach, FL), 248-255.

Giraudet, L., Imbert, J.-P., Bérenger, M., Tremblay, S., and Causse, M. (2016). The neuroergonomic evaluation of human machine interface design in air traffic control using behavioral and EEG/erp measures. Behav. Brain Res. 300:186. doi: 10.1016/j.bbr.2016.01.003

Gonsalvez, C. J., Gordon, E., Grayson, S., Barry, R. J., Lazzaro, I., and Bahramali, H. (1999). Is the target-to-target interval a critical determinant of p3 amplitude? Psychophysiology 36, 643-654. doi: 10.1111/1469-8986.365 0643

Gonsalvez, C. J., and Polich, J. (2002). P300 amplitude is determined by targetto-target interval. Psychophysiology 39, 388-396. doi: 10.1017/S00485772013 93137

Gordon, S., Lawhern, V., Passaro, A., and McDowell, K. (2015). Informed decomposition of electroencephalographic data. J. Neurosci. Methods 256, 41-55. doi: 10.1016/j.jneumeth.2015.08.019

Gordon, S. M., Jaswa, M., Solon, A. J., and Lawhern, V. J. (2017). "Real world bci: Cross-domain learning and practical applications," in Proceedings of the 2017 ACM Workshop on An Application-oriented Approach to BCI out of the Laboratory, BCIforReal '17 (New York, NY: ACM), 25-28.

Handy, T. C., Soltani, M., and Mangun, G. R. (2001). Perceptual load and visuocortical processing: event-related potentials reveal sensory-level selection. Psychol. Sci. 12, 213-218. doi: 10.1111/1467-9280.00338

Hinton, G., Deng, L., Yu, D., Dahl, G. E., Mohamed, A., Jaitly, N., et al. (2012). Deep neural networks for acoustic modeling in speech recognition: the shared views of four research groups. IEEE Signal Process. Magaz. 29, 82-97. doi: 10.1109/MSP.2012.2205597

Hsu, S.-H., Pion-Tonachini, L., Palmer, J., Miyakoshi, M., Makeig, S., and Jung, T.-P. (2018). Modeling brain dynamic state changes with adaptive mixture independent component analysis. Neuroimage 183, 47-61. doi: 10.1016/j.neuroimage.2018.08.001

Ioffe, S., and Szegedy, C. (2015). Batch normalization: accelerating deep network training by reducing internal covariate shift. arXiv [Reprint]. arXiv: abs/1502.03167.

Jeon, Y.-W., and Polich, J. (2003). Meta-analysis of p300 and schizophrenia: patients, paradigms, and practical implications. Psychophysiology 40, 684-701. doi: 10.1111/1469-8986.00070

Jung, T. P., Makeig, S., Humphries, C., Lee, T. W., McKeown, M. J., Iragui, V., et al. (2000). Removing electroencephalographic artifacts by blind source separation. Psychophysiology 37, 163-178. doi: 10.1111/1469-8986.3720163

Kingma, D. P., and Ba, J. (2014). Adam: A method for stochastic optimization. $\operatorname{arXiV}$ arXiv [Reprint]. arXiv: abs/1412.6980.

Kok, A. (2001). On the utility of p3 amplitude as a measure of processing capacity. Psychophysiology 38, 557-577. doi: 10.1017/S0048577201990559

Lawhern, V. J., Solon, A. J., Waytowich, N. R., Gordon, S. M., Hung, C. P., and Lance, B. J. (2018). EEGnet: a compact convolutional neural network for EEG-based brain-computer interfaces. J. Neural Eng. 15:056013. doi: 10.1088/1741-2552/aace8c

LeCun, Y., Bengio, Y., and Hinton, G. (2015). Deep learning. Nature 521, 436-444 doi: 10.1038/nature14539

Lemm, S., Blankertz, B., Dickhaus, T., and Müller, K.-R. (2011). Introduction to machine learning for brain imaging. Neuroimage 56, 387-399. doi: 10.1016/j.neuroimage.2010.11.004

Manor, R., and Geva, A. (2015). Convolutional neural network for multicategory rapid serial visual presentation bci. Front. Comput. Neurosci. 9:146. doi: 10.3389/fncom.2015.00146

Marathe, A. R., Lawhern, V. J., Wu, D., Slayback, D., and Lance, B. J. (2016). Improved neural signal classification in a rapid serial visual presentation task using active learning. IEEE Trans. Neural Syst. Rehabil. Eng. 24, 333-343. doi: 10.1109/TNSRE.2015.2502323

Marathe, A. R., Ries, A. J., Lawhern, V. J., Lance, B. J., Touryan, J., McDowell, K., et al. (2015). The effect of target and non-target similarity on neural classification performance: a boost from confidence. Front. Neurosci. 9:270. doi: 10.3389/fnins.2015.00270

McCarthy, G., and Donchin, E. (1981). A metric for thought: a comparison of p300 latency and reaction time. Science 211, 77-80. doi: 10.1126/science.7444452

McDaniel, J. R., Gordon, S. M., Solon, A. J., and Lawhern, V. J. (2018). "Analyzing p300 distractors for target reconstruction," in 2018 40th Annual International Conference of the IEEE Engineering in Medicine and Biology Society (EMBC) (Honolulu, HI), 2543-2546.

Montavon, G., Samek, W., and Müller, K.-R. (2018). Methods for interpreting and understanding deep neural networks. Digit. Signal Process. 73, 1-15. doi: $10.1016 /$ j.dsp.2017.10.011

Parra, L. C., Christoforou, C., Gerson, A. C., Dyrholm, M., Luo, A., Wagner, M., et al. (2008). Spatiotemporal linear decoding of brain state. IEEE Signal Process. Magaz. 25, 107-115. doi: 10.1109/MSP.2008.4408447

Parra, L. C., Spence, C. D., Gerson, A. D., and Sajda, P. (2005). Recipes for the linear analysis of EEG. Neuroimage 28, 326-341. doi: 10.1016/j.neuroimage.2005.05.032

Passaro, A. D., Vettel, J. M., McDaniel, J., Lawhern, V., Franaszczuk, P. J., and Gordon, S. M. (2017). A novel method linking neural connectivity to behavioral fluctuations: behavior-regressed connectivity. J. Neurosci. Methods 279, 60-71. doi: 10.1016/j.jneumeth.2017.01.010

Polich, J. (2007). Updating p300: an integrative theory of p3a and p3b. Clin. Neurophysiol. 118, 2128-2148. doi: 10.1016/j.clinph.2007.04.019

Polich, J., and Comerchero, M. D. (2003). P3a from visual stimuli: typicality, task, and topography. Brain Topogr. 15, 141-152. doi: 10.1023/A:10226377 32495

Pratt, N., Willoughby, A., and Swick, D. (2011). Effects of working memory load on visual selective attention: Behavioral and electrophysiological evidence. Front. Hum. Neurosci. 5:57. doi: 10.3389/fnhum.2011.00057

Raymond, J. E., Shapiro, K. L., and Arnell, K. M. (1992). Temporary suppression of visual processing in an rsvp task: an attentional blink? J. Exp. Psychol. Hum. Percept. Perform. 18:849. doi: 10.1037//0096-1523.18.3.849

Ren, Y., and Wu, Y. (2014). "Convolutional deep belief networks for feature extraction of EEG signal," in 2014 International Joint Conference on Neural Networks (IJCNN) (Beijing), 2850-2853. 
Ries, A. J., Touryan, J., Ahrens, B., and Connolly, P. (2016). The impact of task demands on fixation-related brain potentials during guided search. PLOS ONE 11:e0157260. doi: 10.1371/journal.pone.0157260

Roy, Y., Banville, H. J., Albuquerque, I., Gramfort, A., Falk, T. H., and Faubert, J. (2019). Deep learning-based electroencephalography analysis: a systematic review. arXiv [Reprint]. arXiv: abs/1901.05498.

Sakhavi, S., Guan, C., and Yan, S. (2015). "Parallel convolutional-linear neural network for motor imagery classification," in 2015 23rd European Signal Processing Conference (EUSIPCO) (Nice), 2736-2740.

Saproo, S., Faller, J., Shih, V., Sajda, P., Waytowich, N. R., Bohannon, A., et al. (2016). Cortically coupled computing: a new paradigm for synergistic humanmachine interaction. Computer 49, 60-68. doi: 10.1109/MC.2016.294

Schirrmeister, R. T., Springenberg, J. T., Fiederer, L. D. J., Glasstetter, M., Eggensperger, K., Tangermann, M., et al. (2017). Deep learning with convolutional neural networks for EEG decoding and visualization. Hum. Brain Mapp. 38, 5391-5420. doi: 10.1002/hbm.23730

Schmidhuber, J. (2014). Deep learning in neural networks: an overview. arXiv [Reprint]. arXiv: abs/1404.7828.

Shamwell, J., Lee, H., Kwon, H., Marathe, A., Lawhern, V., and Nothwang, W. (2016). Single-trial EEG rsvp classification using convolutional neural networks. Proc. SPIE 9836, 1-10. doi: 10.1117/12.2224172

Shrikumar, A., Greenside, P., and Kundaje, A. (2017). Learning important features through propagating activation differences. arXiv [Reprint]. arXiv: abs/1704.02685

Solon, A., Gordon, S., McDaniel, J., and Lawhern, V. (2018). "Collaborative braincomputer interface for human interest detection in complex and dynamic settings," in 2018 IEEE International Conference on Systems, Man, and Cybernetics (SMC) (Miyazaki), 970-975.

Srivastava, N., Hinton, G., Krizhevsky, A., Sutskever, I., and Salakhutdinov, R. (2014). Dropout: a simple way to prevent neural networks from overfitting. J. Mach. Learn. Res. 15, 1929-1958. Available online at: http://jmlr.org/papers/ v15/srivastava14a.html

Sturm, I., Lapuschkin, S., Samek, W., and Müller, K.-R. (2016). Interpretable deep neural networks for single-trial EEG classification. J. Neurosci. Methods 274, 141-145. doi: 10.1016/j.jneumeth.2016.10.008

Sutton, S., Braren, M., Zubin, J., and John, E. R. (1965). Evokedpotential correlates of stimulus uncertainty. Science 150, 1187-1188. doi: $10.1126 /$ science.150.3700.1187

Touryan, J., Apker, G., Lance, B. J., Kerick, S. E., Ries, A. J., and McDowell, K. (2014a). Estimating endogenous changes in task performance from EEG. Front. Neurosci. 8:155. doi: 10.3389/fnins.2014.00155

Touryan, J., Marathe, A., and Ries, A. (2014b). P300 variability during target detection in natural images: implications for single-trial classification. J. Vision 14, 195-195. doi: 10.1167/14.10.195 van Erp, J., Lotte, F., and Tangermann, M. (2012). Brain-computer interfaces: beyond medical applications. Computer 45, 26-34. doi: 10.1109/MC.2012.107

Verleger, R. (1997). On the utility of p3 latency as an index of mental chronometry. Psychophysiology 34, 131-156. doi: 10.1111/j.1469-8986.1997.tb02125.x

Verleger, R., Jaśkowski, P., and Wascher, E. (2005). Evidence for an integrative role of $\mathrm{p} 3 \mathrm{~b}$ in linking reaction to perception. J. Psychophysiol. 19, 165-181. doi: 10.1027/0269-8803.19.3.165

Waytowich, N., Lawhern, V. J., Garcia, J. O., Cummings, J., Faller, J., Sajda, P., et al. (2018). Compact convolutional neural networks for classification of asynchronous steady-state visual evoked potentials. J. Neural Eng. 15:066031. doi: 10.1088/1741-2552/aae5d8

Wickens, C., Kramer, A., Vanasse, L., and Donchin, E. (1983). Performance of concurrent tasks: a psychophysiological analysis of the reciprocity of information-processing resources. Science 221, 1080-1082. doi: $10.1126 /$ science.6879207

Woods, D. L., Courchesne, E., Hillyard, S. A., and Galambos, R. (1980). Recovery cycles of event-related potentials in multiple detection tasks. Electroencephalogr. Clin. Neurophysiol. 50, 335-347. doi: 10.1016/0013-4694(80)90001-2

Xiao, J., Hays, J., Ehinger, K. A., Oliva, A., and Torralba, A. (2010). "Sun database: Large-scale scene recognition from abbey to zoo," in 2010 IEEE Computer Society Conference on Computer Vision and Pattern Recognition (San Francisco, CA), 3485-3492.

Zeiler, M. D., and Fergus, R. (2014). "Visualizing and understanding convolutional networks," in Computer Vision - ECCV 2014, eds D. Fleet, T. Pajdla, B. Schiele, and T. Tuytelaars (Cham: Springer International Publishing), 818-833.

Zhou, B., Lapedriza, A., Khosla, A., Oliva, A., and Torralba, A. (2018). Places: a 10 million image database for scene recognition. IEEE Trans. Pattern Anal. Mach. Intell. 40, 1452-1464. doi: 10.1109/TPAMI.2017.2723009

Conflict of Interest Statement: AS, SG, and JM were employed by DCS Corporation.

The remaining authors declare that the research was conducted in the absence of any commercial or financial relationships that could be construed as a potential conflict of interest.

Copyright (c) 2019 Solon, Lawhern, Touryan, McDaniel, Ries and Gordon. This is an open-access article distributed under the terms of the Creative Commons Attribution License (CC BY). The use, distribution or reproduction in other forums is permitted, provided the original author(s) and the copyright owner(s) are credited and that the original publication in this journal is cited, in accordance with accepted academic practice. No use, distribution or reproduction is permitted which does not comply with these terms. 